\title{
Fibroblast growth factor-23 and fetuin-A in black South African children with chronic kidney disease
}

\author{
A Mudi, ${ }^{1,2} \mathrm{MBBS}, \mathrm{PhD}$; C Dickens, ${ }^{2} \mathrm{PhD}$; R Duarte, ${ }^{2} \mathrm{PhD} ; \mathrm{D}$ Ballot, ${ }^{2} \mathrm{MB}$ BCh, $\mathrm{PhD} ; \mathrm{C}$ Levy, ${ }^{2} \mathrm{MB}$ BCh, MMed \\ ${ }^{1}$ Bayero University College of Health Sciences, Kano, Nigeria \\ ${ }^{2}$ Faculty of Health Sciences, University of the Witwatersrand, Johannesburg, South Africa
}

Corresponding author: A Mudi (amudi.pae@buk.edu.ng)

\begin{abstract}
Background. Both fibroblast growth factor-23 (FGF-23) and fetuin-A levels have been implicated in mineral and bone disorders associated with chronic kidney disease (CKD), and several single nucleotide polymorphisms (SNPs) of the fetuin-A gene have also been associated with fetuin-A levels.

Objectives. This study aimed to determine the relationship between FGF-23 and fetuin-A and to determine the role of fetuin-A SNPs with respect to fetuin-A levels and markers of bone mineralisation in black South African children.

Methods. Blood samples from 93 children (5 - 18 years) with various stages of CKD were assessed for C-reactive protein (CRP), calcium, phosphate, parathyroid hormone, 25-hydroxyvitamin D, FGF-23 and fetuin-A levels. Genomic DNA was extracted from whole blood and regions of the fetuin-A gene amplified by polymerase chain reaction. SNPs were genotyped by restriction fragment length polymorphism analysis or by direct sequencing.

Results. The median FGF-23 and fetuin-A levels were $28.9(11.7$ - 147.1) pg/mL and 57.7 (37.7 - 71.8) mg/dL, respectively. A significant negative relationship between fetuin-A and FGF-23 was only observed in the CKD V group $(p=-0.60, p<0.001)$. Plasma FGF-23 levels correlated better with markers of bone mineralisation than fetuin-A. We found significant association of the fetuin-A SNPs rs4918-G and rs2070633-T alleles with log-transformed fetuin-A levels. Serum phosphate and parathyroid hormone levels were also associated with fetuin-A SNPs.

Conclusion. Plasma FGF-23 levels increase with CKD progression while changes in fetuin-A levels are more likely to be observed in children with advanced CKD; and FGF-23 correlated better with markers of bone mineralisation than fetuin-A.
\end{abstract}

S Afr J Child Health 2020;14(3):139-143. https://doi.org/10.7196/SAJCH.2020.v14i3.1701

Fibroblast growth factor-23 (FGF-23), produced by the osteocytes, is a phosphaturic hormone that regulates phosphate levels by suppressing phosphate reabsorption and suppressing 1,25-dihydroxyvitamin D production in the kidneys. ${ }^{[1]}$ This effect is exerted when FGF-23 binds to its receptors alongside Klotho (an FGF-23 co-receptor). ${ }^{[2]}$ Studies have shown that FGF-23 is associated with progression of chronic kidney disease (CKD) as well as various CKD complications such as mineral and bone disorder (MBD), cardiovascular disease (CVD) and mortality. ${ }^{[3,4]}$

Fetuin-A (also known as a2-Heremans-Schmid glycoprotein (AHSG)) is a multifunctional glycoprotein predominantly produced in the liver and has been described as one of the key circulatory inhibitors of calcification. ${ }^{[1,5]}$ Low levels of fetuin-A have been associated with $\mathrm{MBD}$, vascular calcification, arterial stiffness, atherosclerosis and other cardiovascular events. ${ }^{[6,7]}$

A rise in FGF-23 is often observed in response to hyperphosphataemia observed with declining renal function in CKD patients. ${ }^{[8]}$ On the other hand, studies have reported low fetuin-A levels with declining renal function, possibly as a combined result of reduced fetuin-A production in a pro-inflammatory uraemic environment and increased fetuin consumption in a pro-calcific environment. ${ }^{[9,10]}$ Considering the roles of FGF-23 and fetuin-A, it may be assumed that these biomarkers maintain an inverse relationship in patients with CKD.

Fetuin-A gene polymorphisms have been associated with fetuin-A levels, ultimately affecting cardiovascular injury in both CKD and non-CKD patients. Several single nucleotide polymorphisms (SNPs) of the fetuin-A gene have been implicated in this process. ${ }^{[7,11,12]}$ Eight SNPs of interest (rs2248690, rs6787344, rs4831, rs4917, rs4918, rs2070633, rs2070634 and rs2070635) were identified based on their reported relationship with fetuin-A levels and markers of bone mineralisation in both CKD and non-CKD patients. ${ }^{[7,11-14]}$

The present study aimed to determine the relationship between FGF-23 and fetuin-A in children with CKD and to determine the role of fetuin-A SNPs with respect to fetuin-A levels and biochemical parameters in these children.

\section{Methods}

Thirty-two CKD I, 30 CKD II-IV and 31 CKD V (dialysis) black South African children (age 5 - 18) were recruited consecutively over a 12-month period (August 2015 - July 2016). The CKD I group were children with a glomerular filtration (GFR) $>90 \mathrm{~mL} / \mathrm{min} / 1.73 \mathrm{~m}^{2}$ (with either structural abnormalities, or isolated haematuria) with normal blood pressure and no proteinuria; CKD II-IV were those with GFR of 15 - $90 \mathrm{~mL} / \mathrm{min} / 1.73 \mathrm{~m}^{2}$; and CKD V were those children on maintenance haemodialysis and peritoneal dialysis. Children with known congenital heart disease, diabetes mellitus, liver disease, active infection, systemic lupus erythematosus, malignancies and renal transplant were excluded from the study.

Blood samples were drawn for biochemical assay and genomic DNA. Serum creatinine, phosphate, calcium and intact parathyroid hormone (PTH) levels were assayed using the Siemens Advia system. Plasma 25-hydroxyvitamin D or $25(\mathrm{OH}) \mathrm{D}$ was assayed using the ARCHITECT 25(OH)D method. Plasma intact FGF-23 was assayed using Human FGF-23 ELISA Kit (Merck Millipore, USA). Plasma AHSG level was assayed using EDI Human Fetuin-A ELISA kit (EPITOPE Diagnostics, CA, USA). 
GFR was estimated by use of the modified Schwartz formula. ${ }^{[15]}$ Hyperphosphataemia, hypocalcaemia, elevated calcium product and elevated alkaline phosphatase were defined based on age according to the Kidney Disease Outcomes Quality Initiative (KDOQI) and the Kidney Disease Improving Global Outcomes (KDIGO) clinical practice guidelines for MBD in children with $\mathrm{CKD} \cdot{ }^{[16,17]}$ Hyperparathyroidism was defined as PTH levels above laboratory normal limit $(>7.6 \mathrm{pmol} / \mathrm{L})$ in pre-dialysis patients and above nine times the upper normal limit $(>68.4 \mathrm{pmol} / \mathrm{L})$ in dialysis patients as recommended by KDIGO ${ }^{[17]}$ Low $25(\mathrm{OH}) \mathrm{D}$ was defined as $<30 \mathrm{ng} / \mathrm{mL}$ and elevated C-reactive protein (CRP) as $>10 \mathrm{mg} / \mathrm{L}^{[16,18]}$

Genomic DNA was extracted using the automated Maxwell platform and commercially available Maxwell DNA purification kits (Promega Corp., USA). DNA concentrations were determined by the NanoDrop 2000 spectrophotometer (Thermo Scientific, USA), and the DNA samples stored at $-80^{\circ} \mathrm{C}$. Regions of the AHSG gene covering the SNPs of interest (rs2248690, rs6787344, rs4831, rs4917, rs4918, rs2070633, rs2070634 and rs2070635) were amplified by polymerase chain reaction (PCR) on the MJ Mini Thermal cycler (Bio-Rad, USA). Primers were designed using the IDT PrimerQuest software (https://eu.idtdna.com/PrimerQuest/Home/Index) with sequences and product lengths shown in Table 1. For SNPs rs2248690 and rs6787344, primers were modified to induce a restriction site for restriction fragment length polymorphism (RFLP) analysis. The PCR amplification was carried out using the KAPA2G Robust HotStart ReadyMix PCR Kit using $50 \mathrm{ng}$ DNA and $1.25 \mu \mathrm{L}$ of each of the forward and reverse primers and the recommended thermocycling conditions.

Genotypes for rs2248690, rs6787344, rs4831, rs4917 and rs4918 in fetuin-A were determined by RFLP. Samples were incubated with their respective restriction enzymes overnight at $37^{\circ} \mathrm{C}$ (Table 1). To prevent evaporation, each reaction was overlaid with $15 \mu \mathrm{L}$ of mineral oil. The following day, the reactions were terminated by adding an EDTA-containing gel dye. Fragments were resolved on $10 \%$ polyacrylamide gels and visualised using a Gel Doc EZ imager (Bio-Rad Systems, USA). Three closely positioned SNPs (rs2070633, rs2070634 and rs2070635) were genotyped by direct sequencing at a private laboratory (Inqaba Biotech, SA).
The study was approved by the University of the Witwatersrand Human Research Ethics Committee (Protocol M150312). Written consent was obtained for each parent/guardian of participating children, with assent being obtained from children $>8$ years old.

\section{Data analysis}

All data were collected and managed using the Research Electronic Data Capture (REDCap) tool ${ }^{[19]}$ and STATA 13.1 (Stata Corp., USA) used for the analyses. Continuous variables from the biochemical parameters were described using means (standard deviations) for data normally distributed, and medians (ranges or interquartile ranges (IQR)) used for skewed data. Categorical variables were presented as proportions. Statistical significance in proportions was tested for using $\chi^{2}$ tests or Fisher's exact test where appropriate. Mean/median values of the different groups were compared using anlaysis of variance (ANOVA) or the KruskalWallis test, depending on distribution of the data. To compensate for multiple testing, Bonferroni type correction was used to adjust for significant levels for the biochemical parameters as appropriate.

Genotype frequencies for each SNP distribution were calculated and tested for Hardy-Weinberg equilibrium. In addition to Fisher's exact test or the $\chi^{2}$ test, the trend test of association was used to determine significant difference in the distribution of the genotypes in the CKD groups. Median values of fetuin-A for each of the different SNP genotypes were compared using Kruskal-Wallis tests. To test for relationships between SNPs and fetuin-A levels and markers of mineral bone disease, linear regression was used. Skewed data were log-transformed before inclusion into the regression model. Only the allele and not the genotype patterns were used to determine these relationships owing to low numbers in some of the genotype categories. A $p$-value $<0.05$ was regarded as statistically significant for all analyses.

\section{Results}

The overall mean age of the patients was 10.7 (3.6) years, with a male:female ratio of 2.3:1. The biochemical parameters of the patients are shown in Table 2. The CKD V group had the highest proportion of abnormal parameters.

\section{Table 1. Primers and product lengths for the different SNPs}

\begin{tabular}{|c|c|c|c|c|c|}
\hline \multirow[b]{2}{*}{ SNP } & \multirow[b]{2}{*}{ Primers } & \multirow[b]{2}{*}{ PCR product } & \multirow{2}{*}{$\begin{array}{l}\text { Restriction } \\
\text { enzyme }\end{array}$} & \multicolumn{2}{|c|}{$\begin{array}{c}\text { RFLP allelic } \\
\text { discrimination }\end{array}$} \\
\hline & & & & Allele & Size (bp) \\
\hline \multirow[t]{2}{*}{ rs2248690 } & Fwd: 5' - GAA CCC AGA GCT GTG TCA TA - 3' & $150 \mathrm{bp}$ & NdeI & A & 150 \\
\hline & Rev: 5' - TCC TTC TCC AGA CCT CAC T - 3' & & & $\mathrm{T}$ & 132 and 18 \\
\hline \multirow[t]{2}{*}{ rs6787344 } & Fwd: $5^{\prime}$ - TAC CGA GGT AAG GAG GGA TTG - 3' & $145 \mathrm{bp}$ & $B s a \mathrm{I}$ & $\mathrm{C}$ & 147 \\
\hline & Rev: 5' - CCT TAA AAT AGA TTG GCT AGG GAGA - 3' & & & G & 125 and 20 \\
\hline \multirow[t]{2}{*}{ rs 4831} & Fwd: 5' - GGC AGG CTC CAA CAG ATA AA - 3' & $361 \mathrm{bp}$ & $P v u \mathrm{II}$ & $\mathrm{C}$ & 361 \\
\hline & Rev: 5' - CAT AGA CAG CAG GTC CAC TTAC - 3' & & & G & 199 and 162 \\
\hline \multirow[t]{2}{*}{ rs4917 } & Fwd: 5' - TCT CTG TGG GCA GCA ATA TG - 3' & $284 \mathrm{bp}$ & NlaIII & $\mathrm{C}$ & 284 \\
\hline & Rev: 5' - GGA GGG AAA GGC ATA GCT AAA - 3' & & & $\mathrm{T}$ & 202 and 82 \\
\hline \multirow[t]{2}{*}{ rs4918 } & Fwd: 5' - GGG AGG AGG AAG CAA ACT AAC - 3' & $264 \mathrm{bp}$ & SacI & $\mathrm{C}$ & 264 \\
\hline & Rev: 5' - CAA TGA GAC CAC ACC CAT GAA - 3' & & & G & 209 and 55 \\
\hline $\begin{array}{l}\text { rs2070633, } \\
\text { rs2070634 }\end{array}$ & Fwd: 5' - GCT CTA TGA AAC AGG TGG AAG A - 3' & $439 \mathrm{bp}$ & - & - & - \\
\hline and rs2070635 & Rev: 5' - GGG CTG AGA AGA GTA CAT GAA A - 3' & & & & \\
\hline
\end{tabular}


The median (IQR) FGF-23 and fetuin-A levels were 28.9 (11.7 - 147.1) pg/mL and 57.7 (37.7 - 71.8) mg/dL respectively. Both FGF-23 and fetuin-A levels varied between the different CKD groups (Table 2). Median plasma levels of FGF-23 were lowest in the CKD I group and highest in the CKD V group and differed significantly between the three groups $(p<0.001)$. Fetuin-A levels were almost similar in the CKD I and CKD II - IV groups but much lower in the dialysis-dependant group although the range was wider $(p=0.006)$.

There was no significant linear relationship between total fetuin-A and FGF-23 levels $(\rho=-0.18, p=0.088)$, but a sub-group analysis showed a significant negative relationship between fetuin-A and FGF23 in the CKD V group $(\rho=-0.60, p<0.001)$. Plasma FGF-23 levels correlated better with markers of bone mineralisation than fetuin-A, and no correlation was observed between fetuin-A and CRP (a marker of inflammation) (Table 3).

Eight SNPs were analysed: rs2248690, rs6787344, rs4831, rs4917, rs4918, rs2070633, rs2070634 and rs2070635. Four of these SNPs (rs2248690, rs6787344, rs4918 and rs2070633) did not follow the Hardy-Weinberg law $(p \leq 0.05)$ and were not excluded from the analysis consequent to the small sample size (Table 4).

We found no significant difference in fetuin-A levels in the different SNP genotype distributions, but we found significant association between log-transformed fetuin-A levels and the rs4918 G-allele compared with the rs4918 C-allele ( $p=0.046)$ and the rs2070633 T-allele when compared with the rs2070633 C-allele ( $p=0.015$ ) (Table 5).

Markers of MBD, such as phosphate and PTH levels, were associated with fetuin-A SNPs. The rs6787344 G-allele was significantly associated with phosphate levels (0.042), and the rs4918 G-allele with PTH ( $p=0.044)$ (Table 5).

\section{Discussion}

This comparative study demonstrates changes in the levels of fetuin-A and FGF-23 seen in the different spectra of CKD in black South African children. As expected, given the roles of these two biomarkers in CKD-MBD,${ }^{[8,20]}$ FGF-23 levels showed a linear increase with progression of disease while fetuin-A levels varied across the disease spectrum but did not decrease linearly with disease progression. We identified a strong negative relationship between fetuin-A and FGF-23 in the dialysis group. It is interesting to observe that we did not demonstrate a similar relationship in the other CKD groups, which seems to suggest that, although changes of CKD-MBD may be seen in early CKD, ${ }^{[21,22]}$ a clearer relationship between these two biomarkers is only seen in advanced disease. Our study also showed that FGF- 23 correlated better with markers of bone mineralisation than with fetuin-A.

The near-similar levels of fetuin-A in the CKD I and CKD II-IV groups despite advancing disease and the wider range in the dialysis group cannot be attributed solely to disease progression; other factors such as fetuin-A gene polymorphisms might have contributed to these differences.

Previous studies have reported a link between various fetuin-A SNPs and fetuin-A levels, with the most widely reported SNPs

Table 3. Fetuin-A and FGF-23 association with markers of inflammation and bone mineralisation

\begin{tabular}{lll}
\hline & $\begin{array}{l}\text { FGF-23 } \\
\text { correlation } \\
(p \text {-value })\end{array}$ & $\begin{array}{l}\text { Fetuin A } \\
\text { correlation } \\
(\boldsymbol{p} \text {-value })\end{array}$ \\
\hline CRP $(\mathrm{mg} / \mathrm{L})$ & $0.082(0.435)$ & $-0.165(0.113)$ \\
Calcium $(\mathrm{mmol} / \mathrm{L})$ & $-0.258(0.012)$ & $0.129(0.219)$ \\
Phosphate $(\mathrm{mmol} / \mathrm{L})$ & $0.192(0.065)$ & $-0.044(0.676)$ \\
Alkaline phosphatase $(\mathrm{U} / \mathrm{L})$ & $-0.125(0.234)$ & $0.193(0.064)$ \\
PTH $(\mathrm{pmol} / \mathrm{L})$ & $0.485(<0.001)$ & $-0.253(0.015)$ \\
25(OH)D $(\mathrm{ng} / \mathrm{mL})$ & $-0.370(<0.001)$ & $0.103(0.328)$
\end{tabular}

$\mathrm{CRP}=\mathrm{C}$-reactive protein; $\mathrm{PTH}=$ parathyroid hormone

Table 2. Biochemical parameters of the patients

\begin{tabular}{|c|c|c|c|c|}
\hline Patient parameters & CKD I $(n=32), n(\%)^{*}$ & CKD II-IV $(n=30), n(\%)^{*}$ & Dialysis CKD $(n=31), n(\%)^{*}$ & $p$-value ${ }^{\dagger}$ \\
\hline Age, years ${ }^{\ddagger}$ & $9.5(2.9)$ & $11.0(3.6)$ & $11.6(4.0)$ & 0.051 \\
\hline $\operatorname{Sex}(M: F)$ & $23: 9$ & $24: 6$ & $18: 13$ & 0.167 \\
\hline $\mathrm{CRP}, \mathrm{mg} / \mathrm{L}^{\S}$ & $10(-)$ & $10(10-61)$ & $10(10-62)$ & 0.024 \\
\hline Elevated CRP $(\mathrm{Y} / \mathrm{N})$ & $0 / 32$ & $4 / 26$ & $7 / 24$ & 0.008 \\
\hline Calcium, $\mathrm{mmol} / \mathrm{L}^{\S}$ & $2.32(2.22-2.38)$ & $2.32(2.21-2.37)$ & $2.22(1.99-2.35)$ & 0.005 \\
\hline Hypocalcaemia $(\mathrm{Y} / \mathrm{N})$ & $17 / 15$ & $15 / 15$ & $18 / 13$ & 0.816 \\
\hline Phosphate, $\mathrm{mmol} / \mathrm{L}^{\ddagger}$ & $1.47(0.26)$ & $1.42(0.25)$ & $1.55(0.49)$ & 0.333 \\
\hline Hyperphosphataemia $(\mathrm{Y} / \mathrm{N})$ & $4 / 28$ & $4 / 26$ & $12 / 19$ & 0.023 \\
\hline $\mathrm{CaXP}^{\ddagger}$ & $3.42(0.65)$ & $3.23(0.52)$ & $3.35(1.28)$ & 0.722 \\
\hline Increased CaXP & 0 & 0 & $7 / 24$ & - \\
\hline Alkaline phosphatase, $\mathrm{U} / \mathrm{L}^{\S}$ & $249(209-333)$ & $263(199-310)$ & $252(184-517)$ & 0.669 \\
\hline Increased alkaline phosphatase $(\mathrm{Y} / \mathrm{N})$ & $4 / 28$ & $8 / 22$ & $16 / 15$ & 0.003 \\
\hline $\mathrm{PTH}, \mathrm{pmol} / \mathrm{L}^{\mathrm{S}}$ & $3.8(2.9-5.4)$ & $6.6(3.8-10.9)$ & $60.1(18.0-157.4)$ & $<0.001^{\prime}$ \\
\hline Increased PTH $(y \mathrm{Y} / \mathrm{N})$ & $4 / 28$ & $13 / 16$ & $16 / 15$ & 0.002 \\
\hline $25(\mathrm{OH}) \mathrm{D}^{\ddagger}$ & $22.7(7.4)$ & $25.5(9.1)$ & $18.1(6.7)$ & $<0.002$ \\
\hline Low $25(\mathrm{OH}) \mathrm{D}(\mathrm{Y} / \mathrm{N})$ & $28 / 4$ & $23 / 7$ & $30 / 1$ & 0.059 \\
\hline FGF-23, pg/mL" & $14.99(1.48-90.02)$ & $19.02(0-219.003)$ & $264.10(2.43-3893.00)$ & $<0.001^{\prime}$ \\
\hline Fetuin-A, mg/dL" & $65.48(11.12-96.56)$ & $56.44(26.58-125.98)$ & $34.20(0.85-225.2)$ & 0.006 \\
\hline
\end{tabular}


Table 4. Distribution of SNPs across the different CKD groups CKD CKD V

\begin{tabular}{|c|c|c|c|c|c|c|}
\hline & & $\begin{array}{l}\text { CKD I } \\
(n=32)\end{array}$ & $\begin{array}{l}\text { II - IV } \\
(n=30)\end{array}$ & $\begin{array}{l}\text { - dialysis } \\
(n=31)\end{array}$ & $p$-value ${ }^{*}$ & $p$-value ${ }^{\dagger}$ \\
\hline \multirow[t]{3}{*}{ rs2248690 } & AA & 15 & 15 & 18 & \multirow{3}{*}{0.866} & \multirow{3}{*}{0.402} \\
\hline & AT & 13 & 10 & 10 & & \\
\hline & $\mathrm{TT}$ & 4 & 5 & 3 & & \\
\hline
\end{tabular}

$\begin{array}{lllll}\text { rs6787344 } & \text { CC } & 4 & 3 & 5\end{array}$

$\begin{array}{llllll}\text { CG } & 28 & 27 & 26 & 0.864 & 0.673\end{array}$

GG $0 \quad 0 \quad 0$

rs4831

$\begin{array}{llllll}\mathrm{CC} & 19 & 15 & 12 & & \\ \mathrm{CG} & 11 & 11 & 16 & 0.481 & 0.121 \\ \mathrm{GG} & 2 & 4 & 3 & & \end{array}$

rs4917

$\begin{array}{lllllll}\text { CC } & 20 & 16 & 19 & & \\ \text { CT } & 11 & 13 & 11 & 0.940 & 0.918 \\ \text { TT } & 1 & 1 & 1 & & \end{array}$

$\begin{array}{rllll}\mathrm{rs} 4918^{\dagger} & \mathrm{CC} & 0 & 0 & 1\end{array}$

CG $\quad 30 \quad 29 \quad 30$

$0.327 \quad 0.038$

GG $2 \quad 0 \quad 0$

$\begin{array}{lllll}\text { rs2070633 } & \text { TT } & 12 & 13 & 17\end{array}$

$\begin{array}{llll}\text { TC } & 10 & 5 & 10 \\ \text { CC } & 9 & 12 & 4\end{array}$

$0.128 \quad 0.135$

$\begin{array}{lllll}\text { rs2070634 } & \text { TT } & 12 & 13 & 16\end{array}$

TG $12 \quad 13 \quad 13$

0.495

0.138

GG $7 \quad 4 \quad 2$

rs2070635

AG 0

28

GG 0

2

0

0.104

1.000

$\mathrm{SNP}=$ single nucleotide polymorphism; $\mathrm{CKD}=$ chronic kidney disease.

*Total sample size $<106$.

${ }^{\dagger}$ Fisher's exact or $\chi^{2}$ test as appropriate.

${ }^{*}$ Trend test for association.

Table 5. Regression for CKD risk factors and individual SNP alleles

\begin{tabular}{clll}
\hline & $\beta$ coefficient & $p$-value & $95 \%$ CI \\
\hline log fetuin-A & & & \\
rs4918_C & -0.40 & 0.568 & $-1.78-0.98$ \\
rs4918_G & 1.94 & 0.046 & $0.03-3.85$ \\
rs2070633_T & -0.55 & 0.015 & $-0.98--0.11$ \\
rs2070633_C & -0.10 & 0.616 & $-0.51-0.30$ \\
Phosphate & & & \\
rs6787344_C & -0.16 & 0.568 & $-0.71-0.39$ \\
rs6787344_G & 0.22 & 0.042 & $0.01-0.43$ \\
log PTH & & & \\
rs4918_C & 1.57 & 0.135 & $-0.50-3.65$ \\
rs4918_G & -2.97 & 0.044 & $-5.86--0.08$
\end{tabular}

$\mathrm{CKD}=$ chronic kidney disease; $\mathrm{SNP}=$ single nucleotide polymorphism;

$\mathrm{CI}=$ confidence interval; $\mathrm{PTH}=$ parathyroid hormone . associated with fetuin-A levels being rs4917, rs4918, rs2248690, rs2070633 and rs2070635. ${ }^{[7,12-14,23-25]}$ In the present study, there was a similar association of the rs4918 and rs2070633 SNPs and fetuin-A levels. The rs4918 G-allele showed a positive association with fetuin-A levels, while the rs2070633 T-allele showed a negative association with fetuin-A levels.

Contrary to what has been previously reported, where having the SNP genotypes such as rs4918 CG or GG genotypes was associated with fetuin-A levels, ${ }^{[7,13,14,23,24]}$ we did not observe a similar trend. This variation may be attributed to the small size of our study group, which affected the distribution of the SNP genotypes and alleles. The younger age of our patients and their genetic makeup might have also contributed to the difference.

There is a dearth of studies that have looked at the association between fetuin-A SNPs and markers of bone mineralisation implicated in vascular injury such as phosphate, calcium-phosphate product and parathyroid hormone (PTH). A study by Osawa et al. ${ }^{[14]}$ reported a significant difference in phosphate levels among fetuin-A genotypes. Our study observed a positive relationship of phosphate levels with the fetuin-A SNP rs6787344 G-allele. Serum PTH levels were also found to be negatively associated with rs4918 G-allele.

\section{Limitations}

The major limitations are the small sample size and the lack of a disease-free control group for comparison.

\section{Conclusion}

In spite of the limitations, the present study was able to demonstrate the relationship between FGF-23 and fetuin-A and the association of fetuin-A SNPs with serum fetuin-A, phosphate and PTH levels in children with CKD. The study suggests that FGF-23 increases with progression of disease while fetuin-A levels did not show a linear relationship with disease progression. It also shows that an inverse relationship between FGF-23 and fetuin-A is more likely to be observed in children with advanced CKD, and that FGF-23 correlates better with markers of bone mineralisation in children when compared with fetuin-A. The study also suggests that children with the rs4918 G-allele and rs2070633 T-allele are more likely to have altered fetuin-A levels.

\section{Declaration. None.}

Acknowledgements. The authors acknowledge the contribution of all team members who provided care for these patients and assisted with the study. Author contributions. AM was involved in the conceptualisation, data collection, analyses and drafting of the manuscript. CD, RD, DB and CL were involved with the conceptualisation, analyses and critical review of the manuscript.

Funding. This work was supported by the Carnegie Corporation New York (AMUCARN); the University of the Witwatersrand Faculty of Health Sciences Seed Funding for Research (00125184381045121105); and the Iris Ellen Hodges Cardiovascular Research Trust (001.410.8438104 PAEDHDG).

Conflicts of interest. None.

1. Shroff R, Long DA, Shanahan C. Mechanistic insights into vascular calcification in CKD. J Am Soc Nephrol 2013;24(2):179-189. https://doi.org/10.1681/ asn.2011121191

2. Nitta K, Nagano N, Tsuchiya K. Fibroblast growth factor 23 /klotho axis in chronic kidney disease. Nephron Clin Pract 2014;128(1-2):1-10. https://doi. org/10.1159/000365787 
3. Scialla JJ, Xie $\mathrm{H}$, Rahman $\mathrm{M}$, et al. Fibroblast growth factor-23 and cardiovascular events in CKD. J Am Soc Nephrol 2014;25(2):349-360. https:// doi.org/10.1681/asn.2013050465

4. Isakova T, Xie H, Yang W, et al. Fibroblast growth factor 23 and risks of mortality and end-stage renal disease in patients with chronic kidney disease. JAMA 2011;305(23):2432-2439. https://doi.org/10.1001/jama.2011.826

5. Westenfeld R, Jahnen-Dechent W, Ketteler M. Vascular calcification and fetuin-A deficiency in chronic kidney disease. Trends Cardiovasc Med 2007;17(4):124-128. https://doi.org/10.1016/j.tcm.2007.02.005

6. Scialla JJ, Kao WH, Crainiceanu C, et al. Biomarkers of vascular calcification and mortality in patients with ESRD. Clin J Am Soc Nephrol 2014;9(4):745755. https://doi.org/10.2215\%2FCJN.05450513

7. Maharem DA, Gomaa SH, El Ghandor MK, et al. Association of serum fetuin-A and fetuin-A gene polymorphism in relation to mineral and bone disorders in patients with chronic kidney disease. Egypt J Med Human Genetics 2013;14(4):337-352. https://doi.org/10.1016/j.ejmhg.2013.07.003

8. Russo D, Battaglia Y. Clinical significance of FGF-23 in patients with CKD. Int J Nephrol 2011;2011:364890. https://doi.org/10.4061/2011/364890

9. Ketteler M, Bongartz P, Westenfeld R, et al. Association of low fetuin-A (AHSG) concentrations in serum with cardiovascular mortality in patients on dialysis: A cross-sectional study. Lancet 2003;361(9360):827-833. https://doi. org/10.1016/s0140-6736(03)12710-9

10. Hermans MM, Brandenburg V, Ketteler M, et al. Association of serum fetuin-A levels with mortality in dialysis patients. Kidney Int 2007;72(2):202-207. https://doi.org/10.1038/sj.ki.5002178

11. Lehtinen AB, Burdon KP, Lewis JP, et al. Association of alpha2-HeremansSchmid glycoprotein polymorphisms with subclinical atherosclerosis. J Clin Endocrinol Metab 2007;92(1):345-352. https://doi.org/10.1210/jc.2006-0429

12. Fisher E, Stefan N, Saar K, et al. Association of AHSG gene polymorphisms with fetuin-A plasma levels and cardiovascular diseases in the EPIC-Potsdam study. Circ Cardiovasc Genet 2009;2(6):607-613. https://doi.org/10.1161/ circgenetics.109.870410

13. Stenvinkel P, Wang K, Qureshi AR, et al. Low fetuin-A levels are associated with cardiovascular death: Impact of variations in the gene encoding fetuin. Kidney Int 2005;67(6):2383-2392. https://doi.org/10.111//j.1523-1755.2005.00345.x

14. Osawa M, Tian W, Horiuchi H, Kaneko M, Umetsu K. Association of alpha2HS glycoprotein (AHSG, fetuin-A) polymorphism with AHSG and phosphate serum levels. Hum Genet 2005;116(3):146-151. https://doi.org/10.1007/ s00439-004-1222-7
15. Schwartz GJ, Munoz A, Schneider MF, et al. New equations to estimate GFR in children with CKD. J Am Soc Nephrol 2009;20(3):629-637. https://doi. org/10.1681\%2FASN.2008030287

16. KDOQI. Clinical practice guidelines for bone metabolism and disease in children with chronic kidney disease. Am J Kidney Dis 2005;46(4, suppl 1):S1S103.

17. KDIGO. Clinical practice guideline for the diagnosis, evaluation, prevention, and treatment of Chronic Kidney Disease-Mineral and Bone Disorder (CKDMBD). Kidney International 2009;76 (suppl 113):S3-S130.

18. McIntyre C, Harper I, Macdougall IC, Raine AE, Williams A, Baker LR. Serum C-reactive protein as a marker for infection and inflammation in regular dialysis patients. Clinical Nephrology 1997;48(6):371-374.

19. Harris PA, Taylor R, Thielke R, Payne J, Gonzalez N, Conde JG. Research electronic data capture (REDCap) - a metadata-driven methodology and workflow process for providing translational research informatics support. J Biomed Inform 2009;42(2):377-381. https://doi.org/10.1016/j.jbi.2008.08.010

20. Schäfer C, Heiss A, Schwarz A, et al. The serum protein $\alpha 2$-Heremans-Schmid glycoprotein/fetuin-A is a systemically acting inhibitor of ectopic calcification. J Clin Invest 2003;112(3):357-366. https://doi.org/10.1172\%2FJCI17202

21. Isakova T, Wahl P, Vargas GS, et al. FGF23, PTH and phosphorus metabolism in the chronic renal insufficiency cohort. Kidney Int 2011;79(12):1370-1378. https://doi.org/10.1038\%2Fki.2011.47

22. Wesseling-Perry K, Pereira RC, Tseng CH, et al. Early skeletal and biochemical alterations in pediatric chronic kidney disease. Clin J Am Soc Nephrol 2012;7(1):146-152. https://doi.org/10.2215/cjn.05940611

23. Roos M, Richart T, Kouznetsova T, et al. Fetuin-A and arterial stiffness in patients with normal kidney function. Regul Pept 2009;154(1-3):39-43. https:// doi.org/10.1016/j.regpep.2008.12.001

24. Marechal C, Schlieper G, Nguyen P, et al. Serum fetuin-A levels are associated with vascular calcifications and predict cardiovascular events in renal transplant recipients. Clin J Am Soc Nephrol 2011;6(5):974-985. https://doi. org/10.2215/cjn.06150710

25. Jensen MK, Bartz TM, Djousse L, et al. Genetically elevated fetuin-A levels, fasting glucose levels, and risk of type 2 diabetes: The cardiovascular health study. Diabetes Care 2013;36(10):3121-3127. https://doi.org/10.2337/dc12-2323

Accepted 4 March 2020. 